\title{
HETEROTOPIC OSSIFICATION FOLLOWING NONCEMENTED HIP REPLACEMENT: A COMPARATIVE STUDY USING MINIMAL INVASIVE SURGERY VS. CONVENTIONAL ANTEROLATERAL APPROACH
}

Piyapong Chinkam-akrapat

*Pakchongnana Hospital, Nakhon Ratchasima, Thailand

\begin{abstract}
Background: A conventional anterolateral approach was previously a remedy for total hip replacement. Currently, an intermuscular approach is relatively safe, provides excellent exposure and causes less soft tissue damage than the traditional approach.

Objective: The study aimed to compare heterotopic ossification (HO) between minimal invasive surgery (MIS) and conventional anterolateral approach among patients having noncemented total hip replacement.

Methods: A retrospective study was conducted among 47 patients (52 sites) with noncemented total hip replacement who were randomly divided in 2 groups. The first group received treatment with MIS whereas the second group received the conventional anterolateral approach. The incidence of $\mathrm{HO}$ was recorded and followed-up for a minimum of 12 months. The demographic data of both groups were analyzed using the chi-square test and the discrete data were analyzed using the chi-square test and Fisher's exact test.

Results: The incidence of HO in the MIS and conventional anterolateral approach group were within 37.9 and $56.5 \%$, respectively. After 12 months of followed-up, the incidence of HO in the MIS group did not significantly differ compared with that of the conventional group $(p=0.291)$. Severe HO was within 13.79 and $8.69 \%$, respectively ( $p=0.682)$ and neither group required further surgery.
\end{abstract}

Conclusion: The MIS group showed a lower incidence of HO than that found in the conventional anterolateral approach group without statistical significance.

Keywords : Heterotopic ossification, Minimal invasive surgery, Conventional anterolateral approach

J Southeast Asian Med Res 2019; 3(1): 18-24.

http://www.jseamed.org

Correspondence to:

Chinkam-akrapat P, Pakchongnana Hospital, Nakhon Ratchasima 30130, Thailand.

E-mail : piyapongchinkam@gmail.com 


\section{Introduction}

The indications of total hip arthroplasty (THA) include osteoarthritis, rheumatoid arthritis, avascular necrosis as well as developmental dysplasia. Progressive innovations include many designs of implants such as cemented, noncemented, resurfaces and reconstructed such as endoprosthesis. On the other hand, various styles of approaches are performed depending on the experiences of surgeons. The goal of surgery is excellent or good result and diminished complications. Serious complications including infection, aseptic loosening, dislocation and heterotopic ossification (HO) can disturb patients'daily life activities. The incidence of HO was 24 to $32 \%$, and mostly asymptomatic even though patients with severe grades had limited motion and painful progression. The hypothesis of $\mathrm{HO}$ could occur as described below. First is the process of reaming the femoral canal before inserting the cemented implants that could contaminate the bone marrow surrounding the operative field. Second, modern noncemented implants require impacted broaching. Third, complications may stem from exposure at the hip abductor area in a muscular man. $\mathrm{Biz}$ et al. reported risk factors of $\mathrm{HO}$ included being male, having a gonarthrosis hip joint or contralateral hip with $\mathrm{HO}$ and using the lateral approach. ${ }^{(1)}$ Using meta-analysis, Zhu et al. showed that being male, using cemented implants, bilateral THA, ankylosed hip and ankylosing spondylitis also constituted risks of $\mathrm{HO}^{(2)}$

The severity of $\mathrm{HO}$ depends on the kind of approach employed, which could vary soft tissue dissection and contribute to different grades of HO. Alijanipour et al. reported the incidence of $\mathrm{HO}$ was higher regarding the direct lateral approach than the direct anterior approach. The incidences were 36.1 and $19.4 \%$, respectively, although without significance concerning high grade $\mathrm{HO}{ }^{(3)}$ Similar results to this study were shown by Kutzner et al. reporting that the incidence of $\mathrm{HO}$ after minimal invasive surgery (MIS) using a modified anterolateral approach with short stem was only $7.8 \%$ with excellent Harris Hip Score. ${ }^{(4)}$ In addition, Tan et al. demonstrated that patients could obtain good abductor strength and function in the early postoperative period of 2 years. ${ }^{(5)}$ The objective of this study was to compare HO between MIS using the conventional anterolateral approach among patients having noncemented total hip replacement.

\section{Methods}

A retrospective study was conducted at Pakchongnana Hospital with approval obtained from the Ethics Committee of the Nakhon Ratchasima Provincial Public Health Office (Identity number $=$ KHE 2018-010). The inclusion criteria comprised patients having of the followings: fractured neck of the femur, end stage of gonarthrosis hip, avascular necrosis of the femoral head, Ficat and Arlet stage IV or severe hip dysplasia. The exclusion criteria included patients having one of the followings: traumatic brain injury, stroke, spinal cord injury, severe injury severity score (ISS), ankylosing spondylitis or diffuse idiopathic skeletal hyperostosis (DISH).

From July 2012 to December 2016, the cemented and noncemented total hip replacements and hemiarthroplasty at Pakchongnana Hospital totaled 92 cases. Of these, noncemented THA comprised 48 cases ( 53 sites). All participants were enrolled in the study ( $\mathrm{n}=53$ sites), and one was lost to follow-up due to death. After that, the patients were randomly divided in two groups depending on the date of treatment. The MIS anterolateral approach participants ( 29 sites) constituted the study group while the conventional anterolateral approach participants (23 sites) constituted the control group. All the study operations were conducted by one surgeon. The appearance of $\mathrm{HO}$ was assessed using Brooker classification, which is a common rating scale to score the extent of ectopic bone formation around the hip joint. This classification defines four levels as shown below.

Class 1: bone islands inside tissue around the hip joints

Class 2: bone spur from the pelvis or proximal femur and bony gap more than $1 \mathrm{~cm}$.

Class 3: bone spur from the pelvis or proximal femur and bony gap less than $1 \mathrm{~cm}$.

Class 4: hip ankylosis

A computerized tomography (CT) scan was employed to evaluate $\mathrm{HO}$ in cases of neurologic injury and acetabulum fracture fixation. ${ }^{(6)}$

The severity of $\mathrm{HO}$ related to decreased range of motion and hip function. After treatment, the initial protocol advised by physical therapists in both groups were isometric exercises followed by increased hip range of motion and 
strengthening exercises. Both groups did not take any nonsteroidal anti-inflammatory drugs (NSAIDs) 10 days for prophylaxis of $\mathrm{HO}$ due to gastrointestinal side effects.

Pelvis $\mathrm{X}$-ray imaging was conducted in the anteroposterior (AP) plain and checked periodically after treatment. After at least 12 months of follow-up, x-ray imaging was reviewed by a radiologist uninvolved in the procedures to avoid inter-observer variation and outcome bias. The data were collected by reviewing medical records and x-ray imaging of 48 participants who had underwent noncemented total hip replacement. Medical records were reviewed for demographic data, i.e., sex, age, side of fracture and $\mathrm{HO}$ grade.

\section{Statistical analysis}

The demographic data of both groups were analyzed using the chi-square test and the discrete data were analyzed using the chi-square test and Fisher's exact test. A p-value lower than 0.05 was considered statistically significant.

\section{Results}

Demographic data of both groups such as sex, age and side of fracture are shown in Table 1. No significant differences were found in the demographic data (sex, age, side of fracture) between the two groups and mean follow-up time was $1.3(0.60)$ years in the MIS group and $2.6(1.50)$ years in the conventional approach group. No statistically significant differences were found in the cause of noncemented THA (hip dysplasia, hypertrophic gonarthrosis, avascular necrosis, femoral neck fracture) in both groups (Table 2). HO of the MIS and conventional anterolateral groups totaled 37.9 and $56.5 \%$, respectively ( $p=0.291$ ) as shown in Table 3. Severe HO in both groups totaled 13.8 and $8.7 \%$, respectively $(p=0.682$ ) (Table 4). Although $\mathrm{HO}$ occurred in the conventional group more than in the MIS group, these results were not statistically significant. Neither of the two groups experienced serious complications nor any infections.

Table1. Demographic data among the MIS and conventional groups

\begin{tabular}{llcc}
\hline Data & $\begin{array}{c}\text { MIS group } \\
(29 \text { cases })\end{array}$ & $\begin{array}{c}\text { Conventional group } \\
(23 \text { cases })\end{array}$ & $p$-value \\
\hline $\begin{array}{l}\text { Sex }(\%) \\
\text { female }\end{array}$ & $12(41.4)$ & $11(47.8)$ & 0.854 \\
male & $17(58.6)$ & $12(52.2)$ & 0.187 \\
Age year (Mean \pm SD) & $54.07(11.64)$ & $49.57(12.56)$ & \\
& & & \\
Side of fracture $(\%)$ & $16(55.2)$ & $14(60.9)$ & 0.896 \\
left & $13(44.8)$ & $9 \quad(39.1)$ & \\
right & & & \\
\hline
\end{tabular}

*The chi-square test: gender, side of fracture

Independent t- test: age 
Table 2. Indication of total hip arthroplasty (THA)

\begin{tabular}{llll} 
& Noncemented & total hip replacement & \\
Indicator & MIS & Conventional & $p$-value \\
& $(29$ cases $)$ & $(23$ cases $)$ & \\
\hline Hip dysplasia (\%) & $9(31.03)$ & $11(47.82)$ & 0.343 \\
Non hip dysplasia (\%) & $20(68.97)$ & $12(52.18)$ & $1.000^{*}$ \\
Hypertrophic gonarthrosis (\%) & $5(17.24)$ & $4(17.39)$ & \\
Non hypertrophic gonarthrosis (\%) & $24(82.76)$ & $19(82.61)$ & $1.000^{*}$ \\
& & & \\
AVN of head femur (\%) & $5(17.24)$ & $4(17.39)$ & 0.287 \\
Non AVN of head femur $(\%)$ & $24(82.76)$ & $19(82.61)$ & \\
Fracture femoral neck of femur $(\%)$ & $10(34.48)$ & $4(17.39)$ & $19(82.61)$ \\
Non fracture femoral neck of femur (\%) & $19(65.52)$ & & \\
\hline
\end{tabular}

The chi-square test

*Fisher's exact test

Table 3. Incidence of heterotopic osssification (HO) between the MIS and conventional approaches

\begin{tabular}{lccc}
\hline & & Indicator & \\
Noncemented THA & HO & Non HO \\
amount(\%) & Amolue \\
\hline MIS & $11(37.9)$ & $18(62.1)$ & \\
$(29$ sites $)$ & & $10(43.5)$ & $0.291^{*}$ \\
Conventional & $13(56.5)$ & & \\
$(23$ sites $)$ & & & \\
\hline
\end{tabular}

*The chi-square test 
Table 4. Incidence of severe heterotopic ossification (HO) (Brooker classification $\geq 3$ ) between the MIS and conventional approaches

Indicator

\begin{tabular}{llll} 
Noncemented THA & $\begin{array}{l}\text { Severe HO } \\
\text { number (\%) }\end{array}$ & $\begin{array}{l}\text { Non severe HO } \\
\text { number (\%) }\end{array}$ & $p$-value \\
\hline MIS approach & $4(13.8)$ & $25(86.2)$ & \\
$(29$ sites $)$ & & $21(91.3)$ & $0.682^{*}$ \\
Conventional approach & $2(8.7)$ & & \\
$(23$ sites $)$ & & \\
\end{tabular}

MIS $=$ Minimal invasive surgery

Fisher's exact test: incidence of severe HO

\section{Discussion}

One of the serious complications related to the selected method of approach is HO. HO involves bone inside soft tissue which occurs from osteoinductive growth factor released from soft tissue injury and induced formation of $\mathrm{HO}$. HO is believed to reach its complete formation after 6 to 12 weeks postoperative and not progress anymore after this period. The limited range motion of the hip joint and pain were found only in severe $\mathrm{HO}(9 \%)$, leading to unsatisfactory outcomes after total hip replacement. ${ }^{(7)}$ This study implied a lower rate of $\mathrm{HO}$ using the MIS (37.9\%) when compared with conventional anterolateral approaches $(56.5 \%)(p=0.291)$ while severe HO totaled 13.8 and $8.7 \%$, respectively $(p=0.682)$. However, results of $\mathrm{HO}$ using the two methods were not statistically significant.

Numerous research works have reported that the posterior approach was associated with a lower rate of periprosthetic ossification than the anterolateral or transtrochanteric approach. Moreover, the amount of soft tissue trauma is recognized as a critical risk factor favoring $\mathrm{HO}$ occurrence. In 2004, Bertin and Rottinger described the anterolateral muscle-sparing minimally invasive THA that passed through the interval between the tensor fascia latae and gluteus medius. The popularity of this approach has increased because it provides the potential for reduced blood loss, reduced soft tissue damage, short hospitalization, faster recovery and especially, excellent exposure of the femoral neck and less trochanter. The safe area was defined regarding the distance between the caudal branch of the superior gluteal nerve (SGN) and the apex of the greater trochanter was $5.47(1.61) \mathrm{cm}^{(8)} \mathrm{SGN}$ injury occurs in hip surgery including overstretching the nerve, while retracting or detaching the muscles, thereby causing abductor weakness and a postoperative limp with a positive Trendelenburg's sign.

Presently, the MIS approach is believed to cause less trauma to the soft tissue. Patients could return to work earlier than using standard approaches; however, the incidence of HO remains controversial. Repantis et al. reported the results of a midterm (4 years) study of both clinical and functional aspects between the MIS and conventional anterolateral approaches of THA did not differ regarding functional outcomes and walking endurance except for postoperative pain without reporting the incidence of $\mathrm{HO}^{(9)}$ Hürlimann et al. also reported highest incidence of $\mathrm{HO}$ in a standard modified anterolateral (STD-Watson-Jones) group (45.2\%) and revealed significant 
difference compared with the AMIS (23.1\%) and the STD-Bauer approaches (14.3\%). However, no statistical significance was observed using the MIS-AL approach $(24.0 \%)^{(10)}$

Those who had acute revision total hip replacement within 3 weeks were at high risk of extensive HO. They needed prophylactic treatment because $14 \%$ of Brooker IV and $42.8 \%$ of unsatisfied severe limited motion were observed. ${ }^{(11)}$ The use of extensive surgical wound lavage $(>3000 \mathrm{~mL})$ could decrease the incidence of HO by 73 to $41 \%$ and no severe class of $\mathrm{HO}$ was observed. ${ }^{(12)}$ Likewise, other high risk groups, i.e., diffuse idiopathic skeletal hyperostosis, hypertrophic osteoarthritis, ankylosing spondylitis and previous occurrence of $\mathrm{HO}$, were treated with radiation after surgery. The results of applying the 700 cGy radiation could significantly decrease $\mathrm{HO}$ more than the 400 cGy with no wound complications. ${ }^{(13)}$ In addition, a short course of indomethacin therapy for 10 days prevented some significant grades of $\mathrm{HO}$ and was effective in reducing the incidence of $\mathrm{HO}$ in about one half to two thirds of cases. However, meta-analysis showed no statistical difference was observed when using selective NSAIDs compared with nonselective NSAIDs groups to reduce the incidence of $\mathrm{HO} .^{(14)}$

Some limitations of the present should be noted. This study employed a retrospective and observational review with involving a small number of cases. However, the sample size was sufficient for statistical analysis. This study showed the outcomes of the comparative study concerning the occurrence of HO between the MIS and conventional anterolateral approaches among patients with noncemented hip replacement.

\section{Acknowledgement}

The authors would like to thank Mr. Sommai Khotchanam for his kind assistance in data analysis of this manuscript.

\section{Potential conflicts of interest}

No conflicts of interest were declared by the author.

\section{References}

1. Biz C, Pavan D, Frizziero A, Baban A, Iacobellis C.
Heterotopic ossification following hip arthroplasty: a comparative radiographic study about its development with the use of three different kinds of implants. J Orthop Surg Res 2015; 10: 176.

2. Zhu Y, Zhang F, Chen W, Zhang Q, Liu S, Zhang Y. Incidence and risk factors for heterotopic ossification after total hip arthroplasty: a meta-analysis. Arch Orthop Trauma Surg 2015; $135:$ 1307-14

3. Alijanipour P, Patel RP, Naik TU, Parvizi J. Heterotopic ossification in primary total hip arthroplasty using the direct anterior vs direct lateral approach. J Arthroplasty 2017; 32: 1323 -7.

4. Kutzner KP, Hechtner M, Pfeil D, Rehbein P, Kovacevic MP, Schneider M, et al. Incidence of heterotopic ossification in minimally invasive short-stem THA using the modified anterolateral approach. Hip Int 2017 ;27:162-8.

5. Tan J, Chen H, Chen C, Liang X, Huang W. The strength and function of hip abductors following anterolateral minimally invasive total hip arthroplasty. Chin J Traumatol 2014;17:73-8.

6. Hug KT, Alton TB, Gee AO. Brooker classification of heterotopic ossification after total hip arthroplasty. Clin Orthop Relat Res 2015; 473: 2154-7.

7. Regis D, Sandri A, Sambugaro E. Incidence of heterotopic ossification after surface and conventional total hip arthroplasty: a comparative study using anterolateral approach and indomethacin prophylaxis. Biomed Res Int 2013.doi: 10.1155/2013/293528.

8. Li B, Zhang B, Ding Z, Liu Y, Dai M. Anterolateral intermuscular approach for type A2 intertrochanteric fractures: a cadaveric study. Int Surg 2015; 100:314-9.

9. Repantis T, Bouras T, Korovessis P. Comparison of minimally invasive approach versus conventional anterolateral approach for total hip arthroplasty: a randomized controlled trial.Eur J Orthop Surg Traumatol 2015; 25: 111- 6 .

10. Hürlimann M, Schiapparelli FF, Rotigliano N, Testa E, Amsler F, Hirschmann MT. Influence of surgical approach on heterotopic ossification after total hip arthroplasty - is minimal invasive better? A case control study. BMC Musculoskelet Disord 2017; 18: 27.

11. Aljurayyan A, Tanzer D, TanzerM. Acute revision hip 
arthroplasty: a previously unrecognized risk factor for heterotopic ossification. Eur J Orthop Surg Traumatol 2016; 26: 183-8.

12. Kantak AP, Shah NN. Extensive surgical wound lavage reduces the incidence and severity of heterotopic ossification in primary total hip replacement: a study of 175 hip replacements. Hip Pelvis 2017; 29: 234- 9.

13 Liu JZ, Frisch NB, Barden RM, Rosenberg AG,
Silverton CD, Galante JO. Heterotopic ossification prophylaxis after total hip arthroplasty: randomized trial of 400 vs 700 cGy. J Arthroplasty 2017; 32: 1328-34. 14. Kan SL, Yang B, Ning GZ, Chen LX, Li YL, Gao SJ, et al. Nonsteroidal anti-inflammatory drugs as prophylaxis for heterotopic ssification after total hip arthroplasty: a systematic review and meta-analysis. Medicine (Baltimore) 2015; 94: 828. 